\title{
Association of lower limb muscle mass and energy expenditure with visceral fat mass in healthy men
}

Shusuke Yagi ${ }^{{ }^{*+}}$, Muneyuki Kadota ${ }^{1 \dagger}$, Ken-ichi Aihara $^{2}$, Koji Nishikawa $^{3}$, Tomoya Hara$^{1}$, Takayuki Ise ${ }^{1}$, Yuka Ueda $^{1}$, Takashi Iwase ${ }^{1}$, Masashi Akaike ${ }^{4}$, Michio Shimabukuro ${ }^{5}$, Shinsuke Katoh ${ }^{3}$ and Masataka Sata ${ }^{1}$

\begin{abstract}
Background: A high-calorie diet and physical inactivity, an imbalance between caloric intake and energy consumption, are major causes of metabolic syndrome (MetS), which manifests as accumulation of visceral fat and insulin resistance. However, the lifestyle-related factors associated with visceral fat mass in healthy men are not fully understood.

Methods: We evaluated visceral fat area (VFA), skeletal muscle mass, caloric intake, and energy expenditure in 67 healthy male participants (mean age, $36.9 \pm 8.8$ years; body mass index $23.4 \pm 2.5 \mathrm{~kg} / \mathrm{m}^{2}$ ).

Results: Multiple regression analysis showed that the total skeletal muscle mass $(P<0.001)$ were negatively and age $(P<0.001)$ were positively associated with VFA. Lower limb muscle mass $(P<0.001)$ was strongly associated with VFA. However, total caloric intake, total energy expenditure, and energy expenditure during exercise were not associated with VFA.

Conclusions: Skeletal muscle mass especially lower limb muscle mass negatively contributes to visceral fat mass in healthy men. Therefore, maintaining lower limb muscular fitness through daily activity may be a useful strategy for controlling visceral obesity and metabolic syndrome.
\end{abstract}

Keywords: Exercise, Skeletal muscle, Metabolic syndrome, Prevention

\section{Introduction}

The imbalance between caloric intake and energy consumption, high-calorie diets and physical inactivity, are major causes of metabolic syndrome (MetS), which manifests as accumulation of visceral fat and insulin resistance [1]. The prevention of MetS is an important issue, as it is a major cause of cardiovascular disease (CVD) $[2,3]$.

Lifestyle intervention including caloric restriction and exercise is the preferred approach to reduce the incidence of MetS [4,5]. Exercise not only increases energy consumption but also improves muscle metabolism through increased glucose uptake in skeletal muscles [6]. In addition, exercise enhances skeletal muscle mass,

\footnotetext{
* Correspondence: syagi@tokushima-u.ac.jp

${ }^{\dagger}$ Equal contributors

'Department of Cardiovascular Medicine, The University of Tokushima Graduate School of Health Biosciences, 3-18-15 Kuramoto-cho, Tokushima 770-8503, Japan

Full list of author information is available at the end of the article
}

suggesting that skeletal muscle mass could be a parameter of exercise duration and intensity. Exercise prevents visceral obesity [7]; however, the relashonship between viseral obesity and skeletal muscle mass remains unclear.

The American Heart Association therefore recommends weight reduction to a BMI of $<25 \mathrm{~kg} / \mathrm{m}^{2}$ ) with exercise duration of at least 30 min performed 5 times a week at moderate intensity [1]; however, it is unclear which exercise should be used and which skeletal muscles should be targeted to effectively reduce visceral fat mass in healthy subjects. Since the prevalence of MetS is increasing worldwide, healthy subjects are potentially at risk of MetS [8-10]. Therefore, it is important to identify the risk factors for visceral fat obesity in healthy subjects to prevent MetS. In order to clarify these issues, we evaluated visceral fat mass, skeletal muscle mass, caloric intake, and energy consumption in healthy Japanese men 
Table 1 Clinical characteristics of subjects

\begin{tabular}{lc}
\hline Variables $(\mathbf{n}=\mathbf{6 7})$ & Mean $\pm \mathbf{S D}$ \\
\hline Age $($ years $)$ & $36.9 \pm 8.8$ \\
\hline BW $(\mathrm{kg})$ & $69.2 \pm 8.3$ \\
\hline BMl $\left(\mathrm{kg} / \mathrm{m}^{2}\right)$ & $23.4 \pm 2.5$ \\
\hline Waist circumference $\left(\mathrm{cm}^{2}\right)$ & $84.2 \pm 7.4$ \\
\hline VFA $\left(\mathrm{cm}^{2}\right)$ & $75.0 \pm 29.5$ \\
\hline VFAl $\left(\mathrm{cm}^{2}\right)$ & $41.0 \pm 14.7$ \\
\hline SFA $\left(\mathrm{cm}^{2}\right)$ & $160.3 \pm 56.9$ \\
\hline SFAl $\left(\mathrm{cm}^{2}\right)$ & $87.6 \pm 27.7$ \\
\hline Fat weight $(\mathrm{kg})$ & $15.1 \pm 5.3$ \\
\hline Fat weight/BW (\%) & $21.5 \pm 5.6$ \\
\hline Skeletal muscle weight & $30.4 \pm 3.0$ \\
\hline Total body $(\mathrm{kg})$ & $44.3 \pm 0.03$ \\
\hline Total body/BW(\%) & $5.8 \pm 0.7$ \\
\hline Upper limbs, $(\mathrm{kg})$ & $8.3 \pm 0.7$ \\
\hline Upper limbs/BW, (\%) & $17.5 \pm 1.9$ \\
\hline Lower limbs (kg) & $25.5 \pm 2.3$ \\
\hline Lower limbs/BW (\%) & $7.1 \pm 0.9$ \\
\hline Truncal muscle (kg) & $10.3 \pm 1.3$
\end{tabular}

BW, body weight; BMI, body mass index; VFA, visceral fat area; VFAl, visceral fat area index; SFA, subcutaneous fat area; SFAl, subcutaneous fat area index.

and identified lifestyle-related factors associated with visceral fat mass.

\section{Methods}

We recruited 67 healthy male volunteers aged between 20 and 85 years (mean age, $36.9 \pm 8.8$ years; body mass index (BMI) $23.4 \pm 2.5 \mathrm{~kg} / \mathrm{m}^{2}$ ). Visceral fat area (VFA) and subcutaneous fat area (SFA) were measured using a fat area analyzer (Dual Scan HDS-2000; Omron, Japan) $[11,12]$. Studies have shown that the correlation coefficient between VFA measured by the fat area analyzer and VFA measured by computed tomography was $\mathrm{r}=0.88(\mathrm{p}<0.001)$ [12]. The repeatability of the fat area analyzer was evaluated by the Bland-Altman plot, which has been described elsewhere [12]. These data indicate that this indirect measurement of VFA has a high correlation coefficient with VFA evaluated by computed tomography and does not involve X-ray exposure. Because VFA and SFA were compared with obesity-related variables, which were adjusted with body size represented by body surface area or body weight, VFA and SFA were indexed with body surface area (BSA) as visceral fat area index (VFAI) and subcutaneous fat area index (SFAI), respectively. Skeletal muscle mass was measured with a body composition analyzer (Inbody $7200^{\circ}$; Biospace, Korea) [13]. Body weight and waist circumstance were measured, and BMI was calculated as an index of obesity.

Energy expenditure and total caloric intake was calculated using a questionnaire for food and exercise frequency. Energy expenditure during exercise was defined as energy consumed during exercise per day. Total energy expenditure was defined as energy consumed for daily activity, which includes energy expenditure during exercise. Total caloric intake, energy expenditure during exercise, and total energy expenditure were assessed for 7 days. These values were then averaged per day $[14,15]$.

The study protocol was approved by the Ethics Committee at the Tokushima University Hospital.

\section{Statistical analysis}

For continuous variables, each value is expressed as the mean \pm SD. Single regression analysis was used to assess the correlation between VFA and obesity-related parameters. The degree of association among independent variables, including VFAI, age, skeletal muscle mass, energy expenditure, caloric intake, and parts of skeletal muscles, was assessed by multiple regression analyses (stepwise regression model). All statistical analyses were performed using SPSS software. Statistical significance was defined as $P<0.05$.
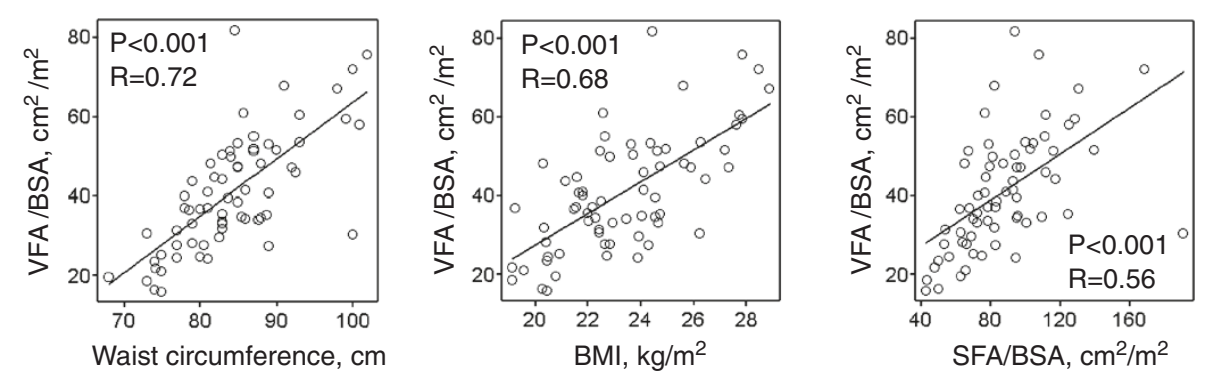

Figure 1 Waist circumference, BMI, and SFA are associated with VFA. VFA: visceral fat area, BMI: body mass index, SFA: subcutaneous fat area, BSA: body surface area. 

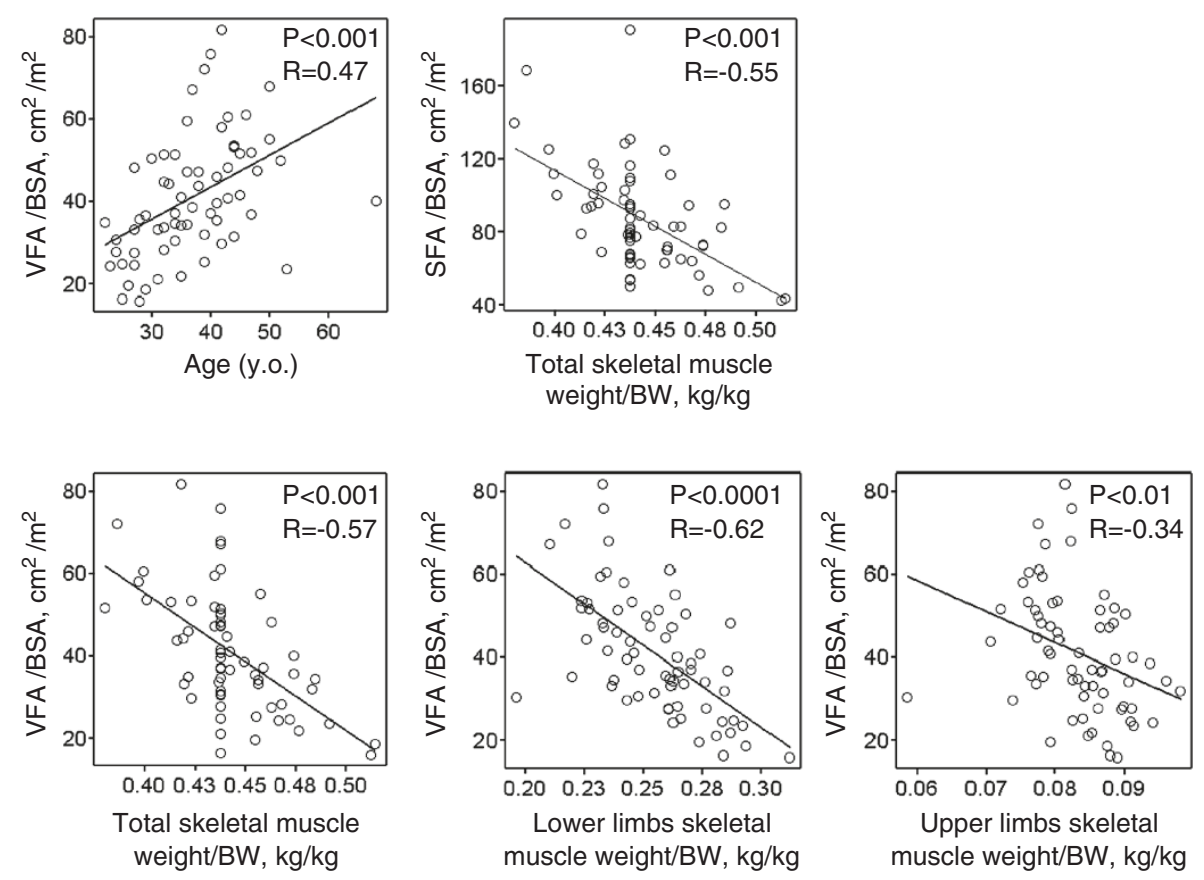

Figure 2 Age is positively associated with increased VFA, while total skeletal muscle mass is negatively associated with both VFA and SFA. Upper and lower skeletal muscle mass are negatively associated with VFA. VFA: visceral fat area, SFA: subcutaneous fat area, BSA: body surface area, BW: body weight.

\section{Results}

\section{Clinical characteristics of subjects}

The clinical characteristics of the subjects are presented in Table 1.

\section{VFAl is inversely associated with skeletal muscle mass and energy expenditure}

The VFAI was positively associated with waist circumference, BMI, SFAI (Figure 1), and age (Figure 2), but was negatively associated with upper, lower and total skeletal muscle mass (Figure 2). The SFAI was negatively associated with total skeletal muscle mass (Figure 2). Neither SFAI nor skeletal muscle mass was associated with age (data not shown).

The VFAI was negatively associated with total energy expenditure and energy expenditure during exercise (Figure 3), but there was no relationship between total caloric intake and VFAI (data not shown).

Stepwise multiple regression analysis showed that total skeletal muscle mass was a negative and age was a
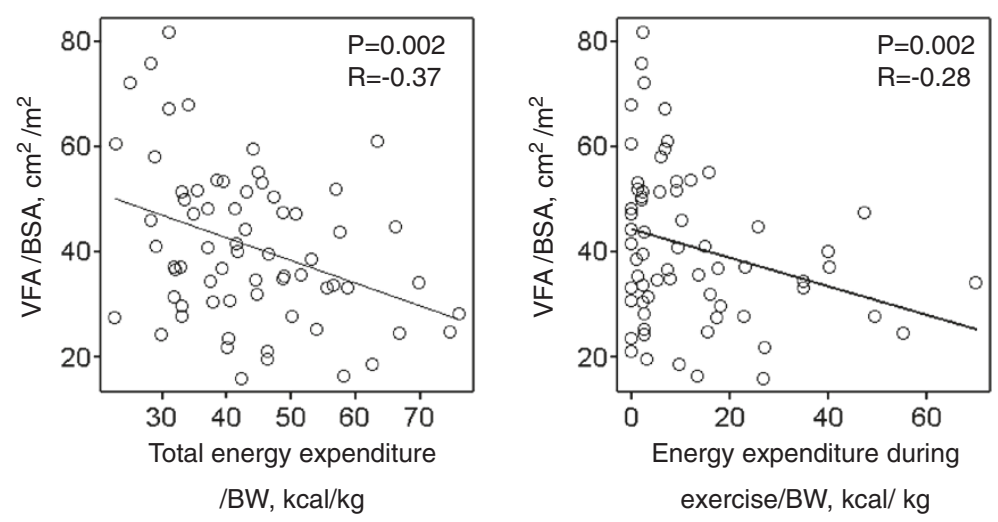

Figure 3 Total energy expenditure for daily-life activity and energy expenditure during exercise are negatively associated with increased VFA. VFA: visceral fat area, BSA: body surface area, BW: body weight. 
Table 2 Multiple regression analysis for determinants of visceral fat area

\begin{tabular}{lcccc}
\hline Variables & Coefficient & 95\% Confidence interval & Standardized coefficient & $\boldsymbol{P}$ value \\
\hline Total skeletal muscle mass & -295 & -404 to -187 & -0.51 & $<0.001$ \\
\hline Age & 0.63 & 0.32 to 0.94 & 0.38 & $<0.001$ \\
\hline
\end{tabular}

$\mathrm{R}^{2}=0.45, P<0.001$

positive determinant of VFAI; however, total caloric intake, total energy expenditure, and energy expenditure during exercise were statistically excluded (Table 2).

VFAI is inversely associated with lower limb skeletal muscle mass

In order to clarify which part of skeletal muscle, including upper limb, lower limb, and truncal skeletal muscle, influences the volume of visceral fat, we performed stepwise multiple regression analysis. Although lower limb skeletal muscle mass was a negative and age was a positive determinant of VFA, upper limb and truncal skeletal muscle mass were statistically excluded (Table 3 ).

\section{Discussion}

The lifestyle-related factors associated with visceral fat mass has been unknown. In this study, we showed that skeletal muscle mass especially lower limb muscle mass are negatively associated with VFAI.

We showed that the VFAI is positively associated with age and negatively associated with skeletal muscle mass. VFA is positively associated with number of metabolic risk factors in the elderly [16], and skeletal muscle mass is inversely associated with age $[17,18]$. However, our data showed that skeletal muscle mass was not associated with age, which is supported by the evidence that muscular strength is inversely associated with the incidence of MetS, independently of age [19]. Although the age outliers might have affected the results (Additional file 1), they nevertheless indicate that the decrease in skeletal muscle mass can be prevented by physical activity.

Decreased skeletal muscle mass leads to physical inactivity [20]. Conversely, physical inactivity leads to decreased skeletal muscle mass [20]. Decreased skeletal muscle mass and strength is associated with increased morality $[21,22]$. Sarcopenic obesity is also associated with hypertension, independent of abdominal obesity
[23]. Increasing skeletal muscle mass and strength via daily exercise may therefore prevent MetS and prolong life span.

In addition, in patients with metabolic syndrome, visceral fat accumulation is accompanied by excess lipid deposition in skeletal muscle, which may contribute to impaired glucose uptake leading to insulin resistance [24]. Improved skeletal muscle functions (including metabolic system) through exercise may contribute to the prevention of MetS [25].

The American Heart Association recommends daily exercise to prevent the accumulation of abdominal fat [1]. Although some subjects exercised in their spare time, our results showed that the association between total energy expenditure during daily activity and VFAI was stronger than the association between energy expenditure during exercise and VFAI. Because the duration of energy expenditure during exercise is relatively short, it may be insufficient for reducing VFA. Therefore, the length of continuous caloric consumption is important for reducing VFA. Enhanced energy expenditure combined with daily exercise is essential for reducing the volume of visceral fat.

Lower limb muscle mass is a determinant of VFAI. Lower limb muscle including the quadriceps forms the largest muscle mass in the body and may therefore contribute to decreased VFA to a greater extent than upper limb or truncal muscle. Lower limb muscle mass and performance in gait are also important because they are associated with reduced mobility, a poor quality of life, CVD, and death [26-28]. Increased physical activity and daily lower body exercise (e.g., brisk walking, cycling, and stair climbing) may be the most useful way to reduce visceral fat and improve mortality. Increased daily activity in young- and middle-aged men may prevent MetS and CVD by decreasing the volume of visceral fat.

In conclusion, skeletal muscle mass especially lower limb muscle mass negatively contributed to VFA in

Table 3 Multiple regression analysis for determinants of visceral fat area

\begin{tabular}{lcccc}
\hline Variables & Coefficient & 95\% Confidence interval & Standardized coefficient & P value \\
\hline Age & 0.52 & 0.20 to 0.84 & 0.31 & -0.53 \\
\hline Lower limb muscle weight & -6.78 & -9.19 to -4.36 & - & $<0.01$ \\
\hline Upper limb muscle weight & - & - & - & - \\
\hline Truncal muscle weight & - & - & -001 \\
\hline $\mathrm{R}^{2}=0.46, P<0.001$. & & &
\end{tabular}


healthy men. Maintaining lower limb muscular fitness through daily exercise may therefore be a useful strategy for controlling visceral obesity and MetS.

\section{Consent}

Informed consent was obtained from the participants for the publication of this report and any accompanying images.

\section{Additional file}

Additional file 1: Age distribution of participants.

\section{Abbreviations}

MetS: Metabolic syndrome; CVD: Cardiovascular disease; VFA: Visceral fat area; SFA: Subcutaneous fat area; BMI: Body mass index; BSA: Body surface area; VFAl: Visceral fat area index; SFAl: Subcutaneous fat area index.

\section{Competing interests}

The authors declare that they have no competing interest.

\section{Authors' contributions}

SY, MK, KN, TH, TIs, YU, and TIw collected data and SY analyzed the data and wrote the manuscript. KA, MA, MSh, SK, and MSa provided the suggestion for this study. All authors read and approved the final manuscript.

\section{Acknowledgments}

This work was supported in part by JSPS KAKENHI Grant Number 25461131.

\section{Author details}

'Department of Cardiovascular Medicine, The University of Tokushima Graduate School of Health Biosciences, 3-18-15 Kuramoto-cho, Tokushima 770-8503, Japan. ${ }^{2}$ Department of Medicine and Bioregulatory Sciences, The University of Tokushima Graduate School of Health Biosciences, Tokushima, Japan. ${ }^{3}$ Department of Rehabilitation, Tokushima University Hospital, Tokushima, Japan. ${ }^{4}$ Department of Medical Education, The University of Tokushima Graduate School of Health Biosciences, Tokushima, Japan. ${ }^{5}$ Department of Cardio-Diabetes Medicine, The University of Tokushima Graduate School of Health Biosciences, Tokushima, Japan.

Received: 20 October 2013 Accepted: 19 February 2014 Published: 26 February 2014

\section{References}

1. Grundy SM, Cleeman II, Daniels SR, Donato KA, Eckel RH, Franklin BA, Gordon DJ, Krauss RM, Savage PJ, Smith SC Jr, Spertus JA, Costa F: Diagnosis and management of the metabolic syndrome: An american heart association/ national heart, lung, and blood institute scientific statement. Circulation 2005, 112:2735-2752

2. Despres JP, Lemieux I: Abdominal obesity and metabolic syndrome. Nature 2006, 444:881-887.

3. Matsuzawa Y: Therapy insight: adipocytokines in metabolic syndrome and related cardiovascular disease. Nat Clin Pract Cardiovasc Med 2006, 3:35-42.

4. Vissers D, Hens W, Taeymans J, Baeyens JP, Poortmans J, Van Gaal L: The effect of exercise on visceral adipose tissue in overweight adults: a systematic review and meta-analysis. PLoS One 2013, 8:e56415.

5. Case CC, Jones PH, Nelson K, O'Brian Smith E, Ballantyne CM: Impact of weight loss on the metabolic syndrome. Diabetes Obes Metab 2002, 4:407-414.

6. Richter EA, Hargreaves M: Exercise, glut4, and skeletal muscle glucose uptake. Physiol Rev 2013, 93:993-1017.

7. Ismail I, Keating SE, Baker MK, Johnson NA: A systematic review and meta-analysis of the effect of aerobic vs. Resistance exercise training on visceral fat. Obes Rev 2012, 13:68-91.

8. Grundy SM: Metabolic syndrome pandemic. Arterioscler Thromb Vasc Biol 2008, 28:629-636.
9. Mozumdar A, Liguori G: Persistent increase of prevalence of metabolic syndrome among U.S. Adults: Nhanes iii to nhanes 1999-2006. Diabetes Care 2011, 34:216-219.

10. Nestel P, Lyu R, Low LP, Sheu WH, Nitiyanant W, Saito I, Tan CE: Metabolic syndrome: recent prevalence in east and southeast asian populations. Asia Pac J Clin Nutr 2007, 16:362-367.

11. Ida M, Hirata M, Odori S, Mori E, Kondo E, Fujikura J, Kusakabe T, Ebihara K, Hosoda K, Nakao K: Early changes of abdominal adiposity detected with weekly dual bioelectrical impedance analysis during calorie restriction. Obesity 2013, 21:E350-E353.

12. Shiga T, Hamaguchi, Oshima Y, Kanai H, Hirata M, Hosoda K, Nakao K: A new simple measurement system of visceral fat accumulation by bioelectrical impedance analysis. IFMBE Proc 2009, 25(VII):338-341.

13. Jensky-Squires NE, Dieli-Conwright CM, Rossuello A, Erceg DN, McCauley S, Schroeder ET: Validity and reliability of body composition analysers in children and adults. Br J Nutr 2008, 100:859-865.

14. Hollowell RP, Willis LH, Slentz CA, Topping JD, Bhakpar M, Kraus WE: Effects of exercise training amount on physical activity energy expenditure. Med Sci Sports Exerc 2009, 41:1640-1644.

15. Reilly JJ, Jackson DM, Montgomery C, Kelly LA, Slater C, Grant S, Paton JY: Total energy expenditure and physical activity in young scottish children: mixed longitudinal study. Lancet 2004, 363:211-212.

16. Nomura K, Eto M, Kojima T, Ogawa S, lijima K, Nakamura T, Araki A, Akishita M, Ouchi Y: Visceral fat accumulation and metabolic risk factor clustering in older adults. J Am Geriatr Soc 2010, 58:1658-1663.

17. Baumgartner RN, Koehler KM, Gallagher D, Romero L, Heymsfield SB, Ross RR, Garry PJ, Lindeman RD: Epidemiology of sarcopenia among the elderly in new mexico. Am J Epidemiol 1998, 147:755-763.

18. Doherty TJ: Invited review: aging and sarcopenia. J Appl Physiol 2003, 95:1717-1727.

19. Jurca R, Lamonte MJ, Barlow CE, Kampert JB, Church TS, Blair SN: Association of muscular strength with incidence of metabolic syndrome in men. Med Sci Sports Exerc 2005, 37:1849-1855.

20. Mancini DM, Walter G, Reichek N, Lenkinski R, McCully KK, Mullen JL, Wilson JR: Contribution of skeletal muscle atrophy to exercise intolerance and altered muscle metabolism in heart failure. Circulation 1992, 85:1364-1373.

21. Wannamethee SG, Shaper AG, Lennon L, Whincup PH: Decreased muscle mass and increased central adiposity are independently related to mortality in older men. Am J Clin Nutr 2007, 86:1339-1346.

22. Ruiz JR, Sui X, Lobelo F, Morrow JR, Jackson AW, Sjostrom M, Blair SN: Association between muscular strength and mortality in men: prospective cohort study. Br Med J 2008, 337:a439.

23. Park SH, Park JH, Song PS, Kim DK, Kim KH, Seol SH, Kim HK, Jang HJ, Lee JG, Park HY, Park J, Shin KJ, Kim DI, Moon YS: Sarcopenic obesity as an independent risk factor of hypertension. J Am Soc Hypertens 2013. in press.

24. Taira S, Shimabukuro M, Higa M, Yabiku K, Kozuka C, Ueda R, Sunagawa S, Ohshiro Y, Doi M, Nanba T, Kawamoto E, Nakayama Y, Nakamura H, Iha T, Nakachi S, Tomoyose T, Ikema T, Yamakawa K, Masuzaki H: Lipid deposition in various sites of the skeletal muscles and liver exhibits a positive correlation with visceral fat accumulation in middle-aged japanese men with metabolic syndrome. Intern Med 2013, 52:1561-1571.

25. Shimabukuro M, Kozuka C, Taira S, Yabiku K, Dagvasumberel M, Ishida M, Matsumoto S, Yagi S, Fukuda D, Yamakawa K, Higa M, Soeki T, Yoshida H, Masuzaki H, Sata M: Ectopic fat deposition and global cardiometabolic risk: new paradigm in cardiovascular medicine. J Med Invest 2013, 60:1-14.

26. Reid KF, Naumova EN, Carabello RJ, Phillips EM, Fielding RA: Lower extremity muscle mass predicts functional performance in mobility-limited elders. J Nutr Health Aging 2008, 12:493-498.

27. Cruz-Jentoft AJ, Baeyens JP, Bauer JM, Boirie Y, Cederholm T, Landi F, Martin FC, Michel JP, Rolland Y, Schneider SM, Topinkova E, Vandewoude M, Zamboni M: Sarcopenia: European consensus on definition and diagnosis. Age Ageing 2010, 39:412-423.

28. Heitmann BL, Frederiksen P: Thigh circumference and risk of heart disease and premature death: prospective cohort study. BMJ 2009, 339:b3292.

doi:10.1186/1758-5996-6-27

Cite this article as: Yagi et al:: Association of lower limb muscle mass and energy expenditure with visceral fat mass in healthy men. Diabetology \& Metabolic Syndrome 2014 6:27. 